\title{
Effects of precursor topology on poly- mer networks simulated with molecular dynamics
}

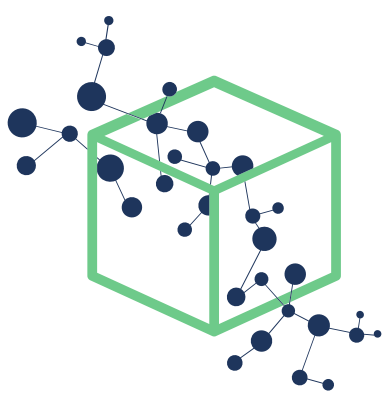

Zhang and Xi (2017)

XI RESEARCH

DOI: 10.1016/j.polymer.2017.03.048

http://xiresearch.org

This document is the accepted manuscript (after peer review) version of an article published in its final form (i.e., the version of record) by Elsevier as

Shimiao Zhang and Li Xi. Effects of precursor topology on polymer networks simulated with molecular dynamics. Polymer, 116:143-152, 2017. doi: 10.1016/j.polymer.2017.03.048

(copyright (C) 2017, Elsevier). The version of record is hosted at

https://dx.doi.org/10.1016/j.polymer.2017.03.048

by the publisher.

The current version is made available under the CC-BY-NC-ND 4.0 license (https://creativecommons. org/licenses/by-nc-nd/4.0/) in accordance with the publisher's policy. Please refer to the publisher's site for additional terms of use.

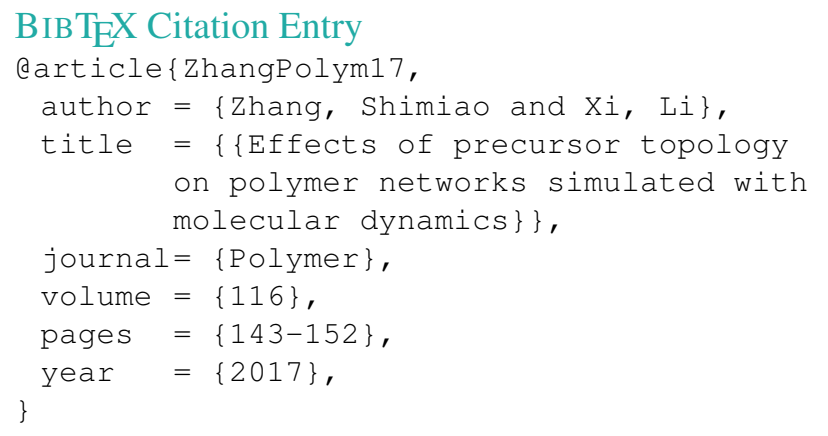




\title{
Effects of precursor topology on polymer networks simulated with molecular dynamics
}

\author{
Shimiao Zhang and $\mathrm{Li} \mathrm{Xi}^{1}$ \\ Department of Chemical Engineering, McMaster University, Hamilton, ON L8S 4L7, Canada
}

\begin{abstract}
Molecular modeling of crosslinked polymers often follows arbitrary pathways for network generation, with different precursor topology from experimental systems. We use coarse-grained molecular simulation to study the effects of precursor choice on the predicted network structure and properties. Three sets of precursors with different molecular architectures are designed such that they would form identical networks at the limit of perfect conversion. Little difference is observed between the resulting networks in typical properties including the radial distribution function, macroscopic statistics of network connectivity, and glass transition behaviors. However, the stress-strain relationship in tensile deformation clearly depends on the formation pathway when compared at the same crosslinking density. The elastic modulus of the network is found to correlate strongly with the number of elastic strands in the network, except at the highly-crosslinked limit where substantial discrepancy is observed between networks from different precursors. Although these final networks contain a similar average density of structural defects, the choice of precursor has significant impact on their spatial distribution, leading to the precursor dependence of their mechanical properties. Uniform defect distribution and fast defect elimination can be achieved by designing precursor units with a proper stoichiometric ratio of different monomers.
\end{abstract}

Keywords: molecular dynamics, polymer networks, cross-linking kinetics, precursor dependence, defect distribution

\section{Introduction}

Network polymers are one of the most important classes of soft materials composed of multiple crosslinked polymer chains which assemble into a percolating threedimensional structure [1 3]. Interconnection of molecular chains by chemical bonds confers many unique properties to these materials, such as improved elasticity and high resistance to heat and organic solvents [4 6]. Because of their superior performance, network polymers have grabbed much attention in both industrial practice and theoretical research. Typical applications include vulcanized rubber and thermosetting polymers (e.g., epoxy resins and phenolic resins) which have been widely used in the manufacturing of tires, parts, and consumer products; they are also a common ingredient for adhesives and coatings, and remain the focus of both experimental and theoretical research [5, 7-[10]. Recent attention in hydrogels, crosslinked hydrophilic polymers swollen by water, further extended their application to the biomedical area [1].

Topologically speaking, the same network can be assembled from different sets of precursors, typically either by reaction between multifunctional monomeric precursors or by connecting linear polymer chains with crosslinkers. Both pathways are regularly used in practice. For instance,

${ }^{1}$ corresponding author, E-mail: xili@mcmaster.ca crosslinked phenolic resins can be synthesized from scratch using phenols and formaldehydes mixed at a proper molar ratio or by curing novolac phenolic resins - uncrosslinked macromolecular precursors - with a crosslinking agent [12. Effects of precursor topology, if present, on the structure and properties of the prepared polymer networks are not well understood.

Development of computer power and molecular simulation techniques over the past three decades have not only significantly advanced our understanding of polymer materials, but also brought us closer to reliable prediction of their properties [13 16. Building molecular models representing realistic microscopic structures of polymers is however not a trivial task [17 20] and the difficulty only multiplies for crosslinked polymers. Ideally, it would be best to have a network formation method that resembles the realistic experimental crosslinking process. It is however prohibitively expensive to simulate the motion and diffusion of all molecules involved for the entire duration of the formation process using chemically realistic full-atom molecular models. Methods for the "virtual" synthesis of polymer networks found in the literature are thus all artificial in this sense. A typical procedure starts with a liquid structure of corresponding monomers, mixed with crosslinker molecules when applicable; new chemical bonds are added between eligible reacting atom pairs within a specified cutoff distance to form a network 21 23. In 
this sing-step approach, optimization techniques are often needed to identify as many potential bonding pairs as possible [24, 25]. The resulting structure normally needs to undergo extensive molecular dynamics runs, often with repeated heating and compression cycles, to relax the stress singularities. Alternatively, these steps can be repeated to form a multi-step crosslinking process, in which new bonds are added in batches and between the bond addition steps the structure is relaxed using molecular dynamics (MD) or Monte Carlo (MC) simulations [26, 27]. To achieve high crosslinking density, where atom motion becomes topologically constrained, it is often necessary to use unrealistically long cutoff distance to push the conversion close to experimental values [22, 27].

Currently there is no standard protocol for the generation of network models. Specific implementation of these methods varies from one research group to another. Meanwhile there is little knowledge of how the choice of formation pathway affects network properties. The only study in this regard is the recent work of Jang et al. 25], which compared a single-step and a multi-step method. Substantial difference was found in the fragment molecular weight between the resulting networks, while all other statistics inspected in the study appear similar.

Model validation is another concern. In most studies, networks in molecular simulation are formed to the same level of crosslinking density as the targeted experimental systems. These models can typically predict volumetric properties such as density and glass transition temperature satisfactorily. However, prediction reliability of many other properties, such as mechanical properties, is not fully examined. As a broader question, if two networks have the same crosslinking density and even the same radial distribution function, does it necessarily ensure their equivalence in all properties? If not, how to detect the discrepancy between a molecular model and its experimental counterpart and which of its predictions are reliable? These questions all remain unexplored.

A realistic reaction-diffusion process for network formation can be simulated at the coarse-grained (CG) level. The earliest CG simulations used a similar approach as the aforementioned full-atom studies, where monomeric precursors are frozen in the space and systematically connected [28, 29]. Seminal work of Kremer, Grest, and coworkers 30 32 pioneered the dynamical approach for network synthesis, which is still widely used today 33 35. Here, diffusion and mobility of polymer chains are fully simulated using MD and crosslinking reaction is assumed to take place when eligible atom pairs fall into a certain cutoff distance. It has been widely used to study the crosslinking kinetics of end-linked polymer networks, i.e., networks formed by connecting the chain ends of linear polymers with multifunctional crosslinkers [30, 33, 35, 37]. Network formation from other precursor architectures has not been reported. CG molecular simulation also provides a powerful tool for understanding the behaviors of network polymers such as their elasticity [38, 39, volume phase transitions [40, and interfacial adhesion behaviors 37, 41. The advantage of CG models in simulating crosslinking kinetics makes it naturally suited for studying the effects of network generation conditions. Using a bond-fluctuation model 42, Gilra et al. 43] observed clear dependence of crosslinking conversion on the cutoff distance. Recent development of systematic coarse-graining methods [44 46] makes it possible to map a network model generated at the CG level, using either MD 47 or MC 48, back to a full-atom representation. Following the same idea, Liu et al. 49] used dissipative particle dynamics (DPD) [50] to model the reaction-diffusion process of the network formation and mapped the generated network structure to the full-atom level for property characterization.

The goal of our study focuses on the effects of formation pathway on the network structure and properties. Almost all full-atom models in the literature were synthesized from monomeric precursors (with the exception of Abbott et al. 23]), it is of interest to understand if crosslinked materials prepared by curing linear polymer precursors can be represented by such models. We design three sets of model precursors in such a way that at the limit of perfect conversion, they would result in identical networks. Our results indicate that precursor topology has non-trivial impact on the network properties. In particular, a precursor design based on the reaction stoichiometry is most efficient in eliminating network defects. Furthermore, differences between these networks are not captured by typical macroscopic structural characterization. These findings show that effects of formation pathway on the molecular model of a polymer network should not be overlooked and new validation protocols need to be developed to adequately vet models for property prediction.

\section{Methodology}

\subsection{Molecular Model and Precursor Systems}

We use the classical bead-spring model of Kremer and Grest [51]: a group of successive repeating units are lumped into one bead (also referred to as one CG "monomer" or atom below), which are connected by finitely-extensible nonlinear elastic (FENE) "bonds" to form a polymer. Interaction between bonded monomers are given by

$$
\begin{aligned}
U_{\mathrm{FENE}}(r)= & -\frac{1}{2} k R_{0}^{2} \ln \left[1-\left(\frac{r}{R_{0}}\right)^{2}\right] \\
& +4 \epsilon\left[\left(\frac{\sigma}{r}\right)^{12}-\left(\frac{\sigma}{r}\right)^{6}+\frac{1}{4}\right]
\end{aligned}
$$

where $r$ is the inter-particle distance. The first term is attractive which models a spring force that diverges at the maximum bond length $R_{0}$ (=1.5 $\sigma$ in our study); the second term is only included for $r<2^{1 / 6}$ which captures the repulsion between beads at short distance. The spring constant $k=30 \epsilon / \sigma^{2}$, which is small enough to allow the use of 


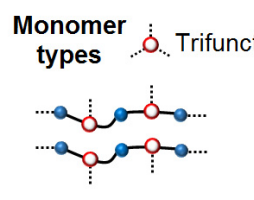

Short chain-Short chain (SS) system

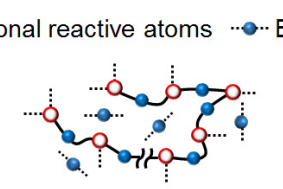

Long chain-Monomer (LM) system (199 beads)
Bifunctional reactive atoms

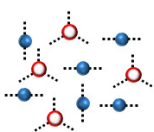

Figure 1: Precursor configurations tested in this study. Solid lines represent existing bonds and dashed lines indicate potential bonding sites.

a relatively large time step while large enough to prevent bonds from cutting through each other 31. The pairwise interaction between non-bonded beads are calculated with the standard Lennard-Jones (LJ) potential [52]

$$
U_{\mathrm{LJ}}(r)=4 \epsilon\left[\left(\frac{\sigma}{r}\right)^{12}-\left(\frac{\sigma}{r}\right)^{6}\right] .
$$

The potential is truncated at $r=2.5 \sigma$ and shifted by a constant to ensure continuity at the cutoff. Results in this study will be reported in standard LJ reduced units: i.e., length is scaled by $\sigma$, energy by $\epsilon$, mass by the bead mass $m$, time by

$$
\tau \equiv \sigma \sqrt{m / \epsilon}
$$

and temperature by $\epsilon / k_{B}, k_{B}$ being the Boltzmann constant.

All simulations are performed with the Largescale Atomic/Molecular Massively Parallel Simulator (LAMMPS) package [53. The equation of motion is integrated by the velocity-Verlet algorithm [52] with a time step of $\Delta t=0.005 \tau$. Periodic boundary conditions are applied in all dimensions.

Precursor configurations tested in this study are shown in Fig. 1. Our model networks are composed of two types of beads with different valences and bonds are only allowed between atoms of opposite types. Three sets of precursors are studied and in all cases we have a 3 : 2 stoichiometric ratio between the total numbers of bifunctional and trifunctional atoms in the system. The monomer/monomer (MM) system models network formation from scratch, starting from monomeric precursors. In the long-chain/monomer (LM) system, linear polymer chains with an alternative sequence of 100 trifunctional and 99 bifunctional monomers are mixed with a complimentary number of free bifunctional atoms to model the curing of polymer melts by crosslinking agents. The third case, the short-chain/short-chain (SS) system, contains five-bead chains; since the $3: 2$ molar ratio is satisfied within the precursor molecules, no additional free atoms are added. This represents a test case in which monomers are "pre-assembled" into oligomeric precursors before crosslinking. The same system size of 50, 000 atoms is used in all cases. For the LM system, this corresponds to 200 linear chains and 10, 200 free bifunctional atoms. The initial thinking was to compare cases with identical atomistic makeup but different precursor sizes, which leads to different precursor diffusion rates and crosslinking kinetics. Our results, however, will show that the precursor topology affects network properties for an entirely different reason.

\subsection{Precursor Equilibration and Network Formation}

Precursor molecules are randomly packed into the simulation box at an atom number density of 0.85 . For linear chains, constituting atoms are added to random positions one by one in the order of the chain sequence. These configurations require further relaxation owing to atom overlapping and for the LM case an unrealistic initial chain conformation. For MM and SS systems, overlaps are removed by a so-called fast push-off step [54, in which pairwise interactions between non-bonded atoms are first replaced with a soft potential

$$
U_{\text {soft }}(r)= \begin{cases}A_{\text {soft }}\left[1+\cos \left(\frac{\pi r}{r_{c}}\right)\right] & r<r_{c} \\ 0 & r \geq r_{c}\end{cases}
$$

with a cut-off distance of $r_{c}=2^{1 / 6} \sigma$. The potential amplitude $A_{\text {soft }}$ is ramped up from $4 \epsilon$ to $200 \epsilon$ over a time period of $10 \tau$. We then switch to the regular LJ potential (Eq. (2) and further equilibrate the configuration using MD (NVT ensemble) for $250 \tau$.

For the LM system, we use instead a DPD push-off method designed for fast equilibration of melt structures of long linear chains [19]. During the equilibration stage, the non-bonded pairwise interaction is set to

$$
U_{\mathrm{DPD}}(r)= \begin{cases}\frac{A_{\mathrm{DPD}}}{2} r_{c}\left(1-\frac{r}{r_{c}}\right) & r<r_{c} \\ 0 & r \geq r_{c}\end{cases}
$$

which is the potential energy corresponding to the conservative force of DPD [50]; the cut-off distance $r_{c}=\sigma$. DPD simulation is run at $T=1 \epsilon / k_{B}$ using a constant $A_{\mathrm{DPD}}=$ $25 k_{B} T$ for $500 \tau ; A_{\mathrm{DPD}}$ is then ramped to $1000 k_{B} T$ over $5.5 \tau$. The system is further equilibrated with the regular LJ potential (Eq. 2p) for $500 \tau$ in an NVT ensemble.

Starting from the equilibrated precursor configuration, network formation is performed with MD in the NVT ensemble. The Nosé-Hoover thermostat [55] is used to maintain $T=1 \epsilon / k_{B}$ and the box size is fixed at $19.45 \sigma$ in all three dimensions, corresponding to an atom density of 0.85 . Distances between atoms of opposite types are checked every 10 time steps and a new FENE bond is created between each eligible reactant pair within a critical reaction radius of $1.0 \sigma$. If at a given step, an atom finds more than one eligible reaction partners, the closest one is chosen. Reactions between atoms of the same type are forbidden during the network formation process.

All simulations in this study are repeated from three independent initial configurations. Unless otherwise noted, results presented below are the averages of the three runs and error bars show the standard errors. 


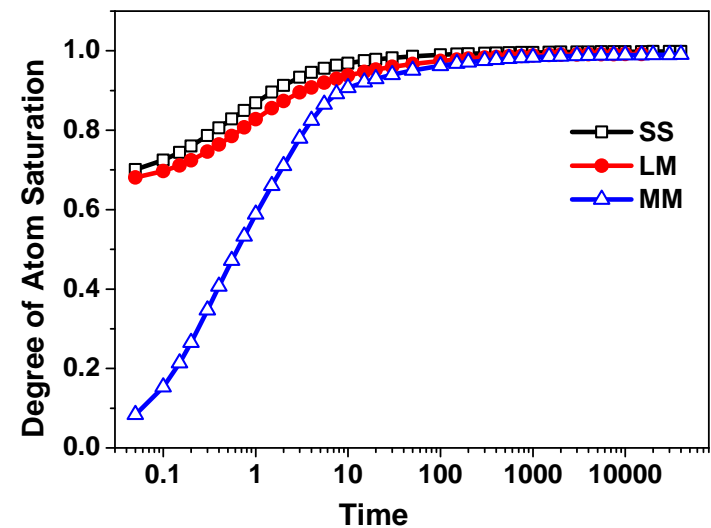

(a)

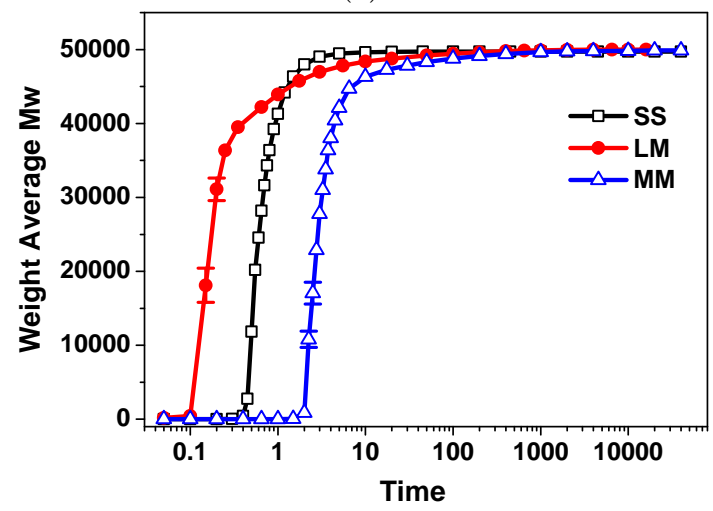

(b)

Figure 2: Time evolution of (a) the degree of atom saturation and (b) weight-averaged molecular weight of SS, LM and MM systems. Error bars smaller than the symbol size are not shown.

\section{Results and Discussion}

\subsection{Network Formation and Structure}

We define the

$$
\begin{aligned}
& \text { degree of atom saturation } \equiv \\
& \frac{\text { number of bonds }}{\text { theoretical maximum number of bonds }}
\end{aligned}
$$

where the denominator is the number of bonds in a perfectly-formed network with all bonding sites fully saturated; for a system size of 50,000 (20,000 of the atoms are trifunctional) this number is 60,000 . This ratio is calculated for all three precursor systems as a universal measurement of polymerization progress and the results are plotted in Fig. 2a. In forming the precursor chains, some bonding sites are taken; thus the number starts from nonzero values for LM and SS systems. All three cases show an initial stage of fast growth until $t \sim 10 \tau$ after which the reaction rate drops down sharply. The final atom saturation degrees are all above $98 \%$. Figure $2 \mathrm{~b}$ shows the evolution of the weight-averaged molecular weight $\left(M_{w}\right)$ during the formation process. As expected, the gelation point, indicated by a sharp increase in the molecular weight, occurs earlier as the chain length of precursors increases. This

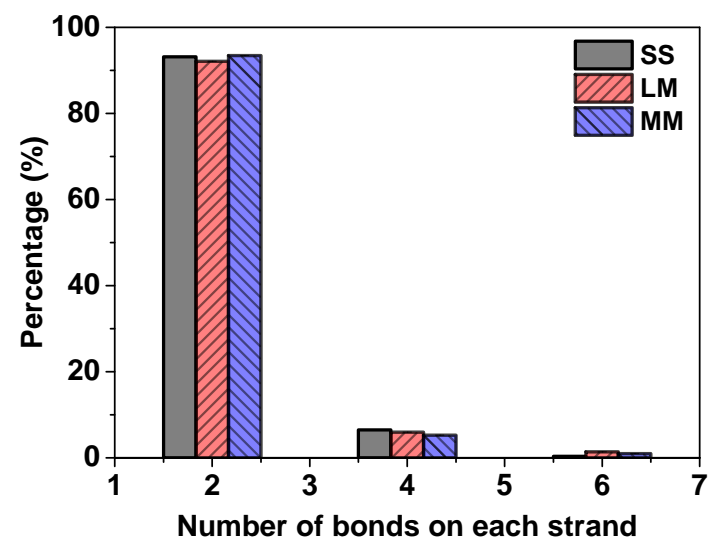

Figure 3: Distribution of elastic-strand lengths - measured by the number of consisting bonds - of the final network images. Error bars are too small to show.

observation stems from the fact that staring from longer polymer chains, it takes less new bonds to form a percolating network. In all three cases, the final $M_{w}$ is close to the limit of fully connected network where $M_{w, \max }=50,000$.

To focus on the precursor effect, we now compare networks at nearly the same crosslinking density, which is measured by the degree of atom saturation. For each process, we select an image with $\approx 99 \%$ atom saturation as a representation of the final product. Structures of these final networks are first characterized in terms of the topology of network connectivity. The elastic part of a network is composed of elastic strands - chain segments whose two ends connect to different junction points - and elastic junctions - atoms where multiple (in our case 3) elastic strands meet. All other chain segments only have one end attached to the network and thus do not contribute to the elasticity. These defects are called dangling chains. (Chain loops are another major type of topological defects often discussed in the literature 56]. However, in our model, a loop can only be formed with a chain starting from and ending in a same trifunctional atom. By that the loop occupies two of its three bonding sites, leaving only one for connection with the network. Therefore, loops can only appear at the end of a dangling chain and thus they are lumped into the same category of defects.)

These topological features are analyzed using the structure searching method of Duering et al. [57, which is based on the "burning method" 58; the results are listed in Table 1. The numbers of elastic junctions and elastic strands are statistically indistinguishable between different precursor systems. The number of dangling chains is only slightly different with the SS case having the least and MM the most. In all three cases, this number is small for the size of the network. In addition, these dangling chains are all short, most of which contain only one atom each. Length distributions of elastic strands are compared in Fig. 3. Note that in our model setup, only trifunctional atoms act as junction points; in the limit of perfectly-formed network, there will be exactly one bifunctional atom between 
Table 1: Structural parameters of the final network images (normalized by the parameters of a perfect network at full conversion)

\begin{tabular}{llllll}
\hline $\begin{array}{l}\text { Precursor } \\
\text { system }\end{array}$ & $M_{w}(\%)^{\mathrm{a}}$ & bonds $(\%)^{\mathrm{a}, \mathrm{c}}$ & $\begin{array}{l}\text { dangling chains } \\
(\%)^{\mathrm{b}}\end{array}$ & $\begin{array}{l}\text { elastic junctions } \\
(\%)^{\mathrm{a}}\end{array}$ & $\begin{array}{l}\text { elastic strands } \\
(\%)^{\mathrm{a}}\end{array}$ \\
\hline SS & $99.42 \pm 0.01$ & $99.17 \pm 0.01$ & $1.82 \pm 0.03$ & $93.52 \pm 0.12$ & $93.52 \pm 0.12$ \\
LM & $99.85 \pm 0.01$ & $98.88 \pm 0.00$ & $2.02 \pm 0.02$ & $93.56 \pm 0.02$ & $93.56 \pm 0.02$ \\
MM & $99.75 \pm 0.06$ & $99.00 \pm 0.00$ & $2.15 \pm 0.06$ & $93.23 \pm 0.10$ & $93.23 \pm 0.10$ \\
\hline
\end{tabular}

a The molecular weight $M_{w}$, number of bonds, number of elastic junctions, and number of elastic strands are normalized by their maximum possible values as in a perfect network (50000, 60000, 20000, and 30000, respectively).

$\mathrm{b}$ The number of dangling chains is normalized by the maximum number of elastic strands as in a perfect network (30000).

c The normalized number of bonds is the same as the degree of atom saturation (eq. 6).

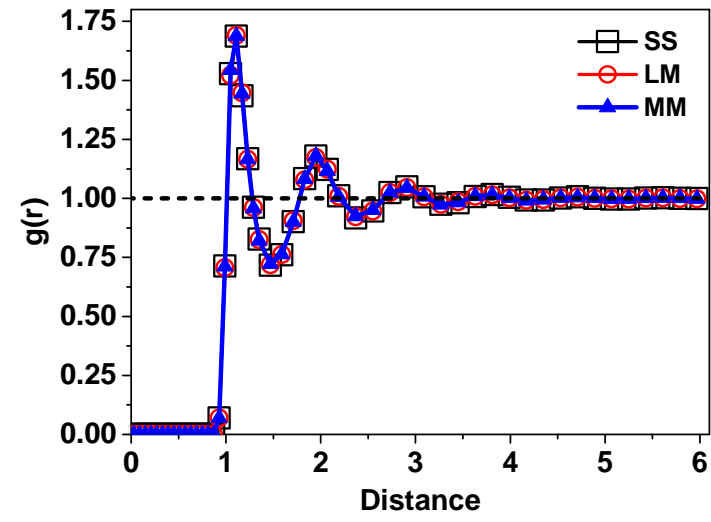

Figure 4: Radial distribution functions of the final network images.

every two neighboring junction points. Therefore, all elastic strands will have the exact same length of 2 bonds. Final networks from different precursors are all close to this limit with over $90 \%$ of the strands containing 2 bonds. A small fraction of 4-bond and 6-bond strands are found.

Radial distribution functions $g(r)$ of the final network images are shown in Fig. 4. No discernible difference is observed between different cases, indicating that the atom arrangement, at least at the average level, is essentially the same. Specific volume during a controlled cooling process is shown in Fig. 5. The selected final structures are first subject to several heating and cooling cycles to remove their thermal history. The system is then cooled at a constant rate of $1.6 \times 10^{-4} \epsilon /\left(k_{B} \tau\right)$ using the Nosé-Hoover thermo- and baro-stats $(P=0)$ 55. The glass transition temperature $T_{g}$ is identified from a sudden change in the slope. For an uncrosslinked melt consisting of 200 199bead linear chains, our $T_{g}=0.435$, which is in excellent agreement with the $T_{g}=0.43$ found by Makke et al. 59] (whose system is closest to ours). It is also within the range of $0.4-0.45$ typically reported for linear chains [60, 61]. For networks, glass transition occurs at $T_{g}=0.479$ regardless of the precursor choice. Higher $T_{g}$ is expected at high crosslinking density owing to the shorter average strand length 62. Not only is there no discernible dependence of $T_{g}$ on the precursor topology, volumetric curves from these three cases are essentially indistinguishable.

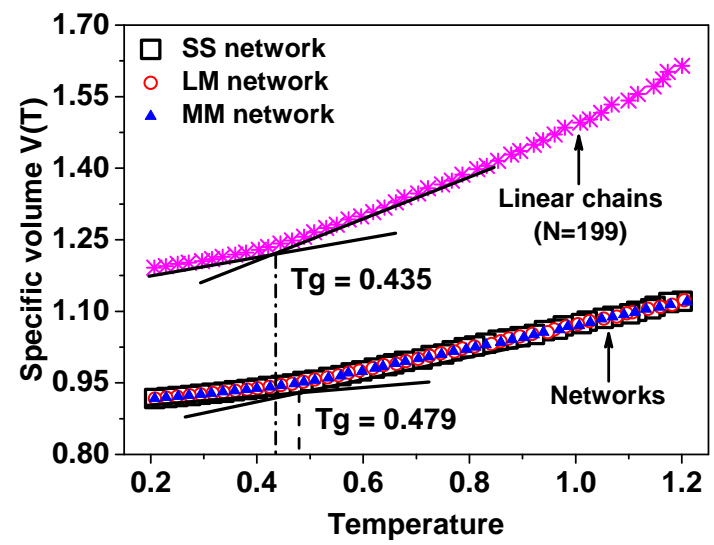

Figure 5: Temperature dependence of specific volume in a controlled cooling process of the final network images.

\subsection{Mechanical Properties and Structural Defects}

Topological parameters and macroscopic properties examined so far showed little lasting effect of the choice of precursors. Mechanical properties of the networks will however reveal a different side of the story. Tensile elongation tests are simulated by deforming the simulation box along one dimension, denoted as $z$, with the timedependent box length set to

$$
L_{z}(t)=L_{z, 0} \exp (\dot{e} t)
$$

and zero pressure maintained in the transversal directions ( $x$ and $y$ ) by the Nosé-Hoover barostat. Here $L_{z, 0}$ is the initial box length and $\dot{\epsilon} \equiv d L /(L d t)$ is the constant strain rate; in our simulation $\dot{\epsilon}=0.0327 \sigma / \tau$. The tensile stress is calculated from the measured pressure difference between the elongation and transversal directions:

$$
s=-P_{z}+\frac{1}{2}\left(P_{x}+P_{y}\right)
$$

which is plotted against the engineering strain

$$
e \equiv \frac{L_{z}-L_{z, 0}}{L_{z, 0}}
$$

in Fig. 6. The qualitative trends observed in the stressstrain curves are typical for polymer networks. Beyond the 


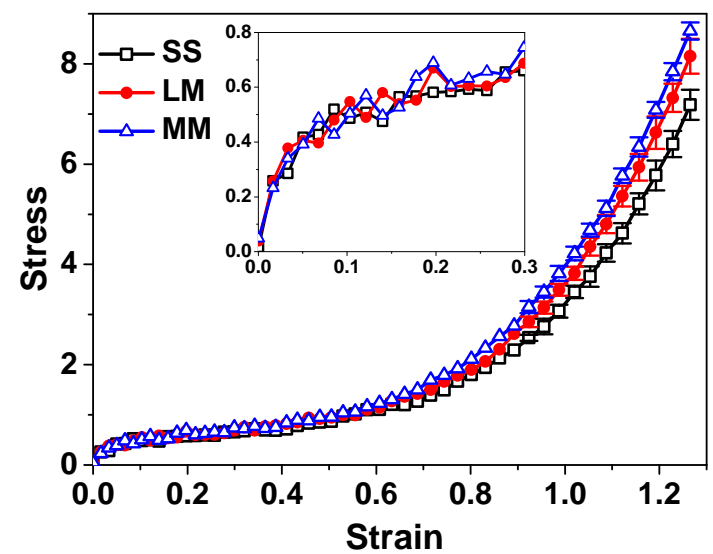

Figure 6: Stress-strain curves of the final network images during tensile deformation; the inset shows a blowup of the small-stain regime. Error bars smaller than the symbol size are not shown.

linear regime, which is only observed for $e$ up to $O(0.01)$, the networks enter the strain-softening regime first before the strain-hardening regime at large strain. Networks from different precursors show no discernible difference at small or moderate deformation. Agreement between different cases is expected at the limit of small strain according to classical theories for rubber elasticity. For example, the affine network model (ANN) predicts that 63

$$
E \propto G=\nu_{\text {strand }} k_{\mathrm{B}} T
$$

where $E$ is the Young's modulus, $G$ is the shear modulus, $\nu_{\text {strand }}$ is the number density of elastic strands, $k_{\mathrm{B}}$ is the Boltzmann constant, and $T$ is temperature. Networks being compared here have nearly the same $\nu_{\text {strand }}$ (see Table. 1) and thus same modulus. However, at the large-strain limit $e \gtrsim 0.7$, where $\mathrm{ANN}$ is no longer applicable, the curves start to separate. The network stiffness increases from the SS to the LM case, with the MM case being the highest. These differences are clearly larger than the statistical uncertainty. Note again that the images compared here are selected at a nearly identical degree of atom saturation (and thus crosslinking density), differences in mechanical response can only originate from the precursor topology. This precursor effect is not reflected in $g(r)$ and specific volume $V(T)$ curves (Figs. 4 \& 5) where data are indistinguishable between the three cases. Since the structural parameters listed in Table. 1 are statistically nearly the same, there must be other structural differences not captured by these measurements.

To understand this precursor dependence, we need to first identify the structural origin of the property differences. In Fig. 7, we take the apparent elastic modulus

$$
E_{\text {app }} \equiv \frac{s}{e}
$$

at the strain of $e=1$ as a measurement of mechanical properties and attempt to find the structural parameter with which it best correlates. Images spanning a broad

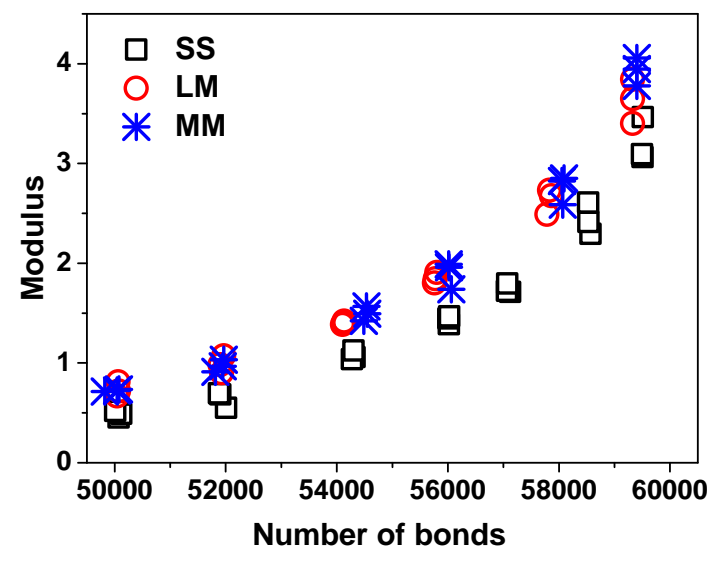

(a)

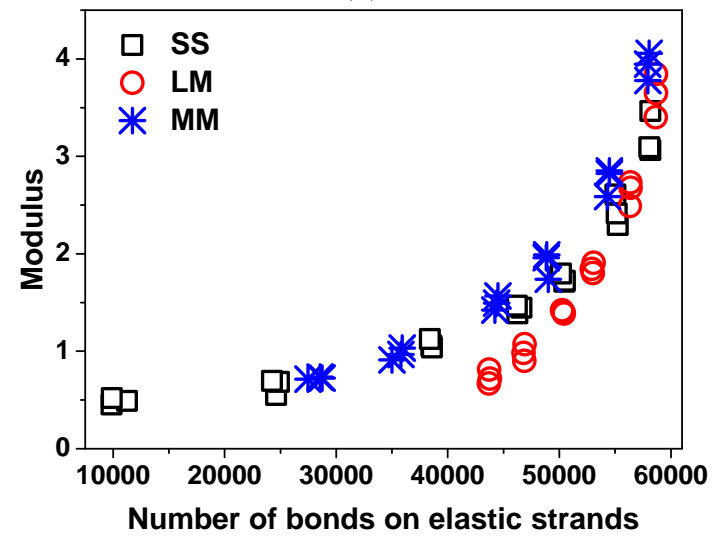

(b)

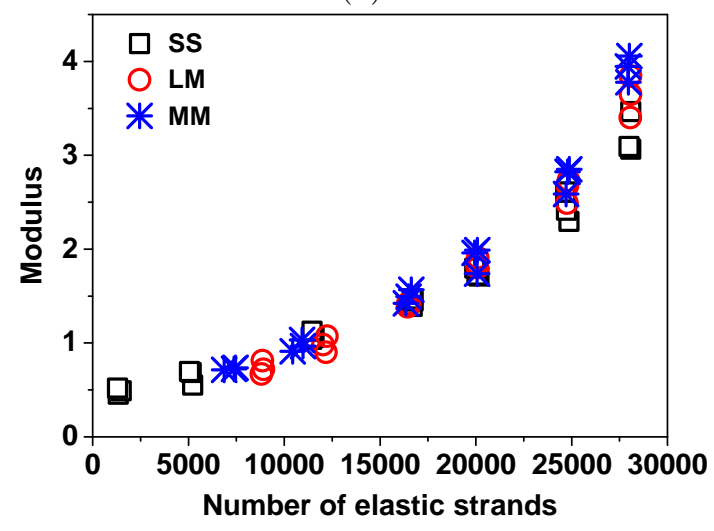

(c)

Figure 7: Correlation between the apparent elastic modulus and the (a) total number of bonds, (b) number of bonds on elastic strands, and (c) number of elastic strands. For each precursor system, results from independent runs are shown as separate points. 


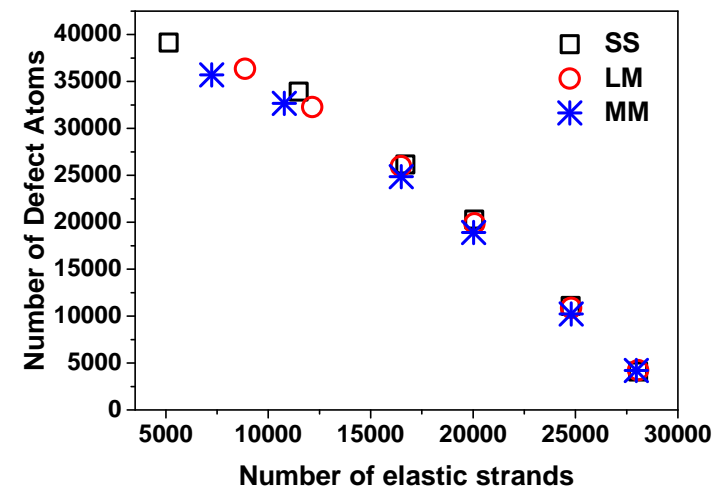

Figure 8: Correlation between the number of atoms in topological defects and the number of elastic strands. Error bars are smaller than the symbol size.

range of polymerization progress (or crosslinking density), from the gel point to nearly full conversion, are included. The total number of bonds (Fig. 7a) does not adequately predict $E_{\text {app }}$; especially, data points of the SS system are systematically lower than the rest. This is not surprising since bonds on structural defects, such as dangling chains or loops, do not carry the stress during deformation and thus do not contribute to the mechanical strength. We then count the number of bonds on elastic strands only (Fig. 7b), which again falls short. Especially, in the intermediate range of crosslinking density, $E_{\text {app }}$ of the LM case is significantly lower than the other two at the same number of elastic-strand bonds. This is due to its unique network formation mechanism: a small number of bonds are sufficient to connect the individual chains into a percolating network, where initially there are on average a large number of bonds separating the elastic junctions (i.e., large elastic strand length); additional crosslinking bonds are then added in between to stitch it into a denser network. By contrast, in the MM and SS cases, networks are generated by grafting small pieces on to growing tree-like clusters before the gel point and it is unlikely to form a long strand without branching. This prompts us to look instead into the number of elastic strands, which as shown in fig. $7 \mathrm{c}$ correlates strongly with $E_{\text {app }}$ for a broad range of crosslinking density. The data points however become scattered at the highly-crosslinked limit (where the number of elastic strands exceeds $83 \%$ of that of a perfect network). Indeed, at least for the final networks, the numbers of elastic strands are statistically the same between different precursor systems (see Table. 1), yet $E_{\text {app }}$ can differ by $\approx 30 \%$ depending on the precurors used. There must be other structural differences to account for the different mechanical properties at this limit.

Topological defects are known to impact the mechanical properties of networks [36, 64] and it is intuitive to expect that their effects would become more pronounced as the network gets close to completion. However, although the number of dangling chains is slightly different between precursor systems (see Table.11), it cannot explain the varying elastic modulus, since dangling chains do not directly contribute to the network elasticity. Indeed, we have repeated the tensile tests with all dangling chains removed and the precursor-dependence is still obvious. Additional types of defects thus need to be examined. As explained above, the only other difference between a perfect and an imperfect network is that the latter contains elastic strands longer than 2 bond lengths. These strands contain at least one unsaturated trifunctional atom in the middle. Atoms on these unsaturated strands are expected to have higher mobility, resulting in different elastic response than the standard 2-bond strands. Hereinafter, atoms on dangling chains and unsaturated strands are both considered defect atoms. As shown in Fig. 8, the total number of defect atoms are closely correlated with the number of elastic strands, especially for highly-crosslinked polymers. Therefore, it cannot account for the precursor dependence of $E_{\text {app }}$ between networks with the same number of elastic strands (see Fig. 7c).

The lack of explanation from domain-average statistics prompts us to look into the spatial distribution of structural characteristics. Figure 9 shows the density distribution of defect atoms in the simulation domain. Here, the contribution of atom $i$ to the density field is modeled with a Gaussian distribution function 65.

$$
\rho_{i}(\boldsymbol{x})=\exp \left(\frac{-\left\|\boldsymbol{x}-\boldsymbol{x}_{\boldsymbol{i}}\right\|^{2}}{2 r_{\text {atom }}^{2}}\right)
$$

where $\boldsymbol{x}_{\boldsymbol{i}}$ is the position of atom $i$ and $r_{\text {atom }} \equiv 0.5 \sigma$ is its radius. Defect atom density is obtain by adding up individual contributions: i.e., $\rho_{\text {defect }}(\boldsymbol{x})=\sum_{i} \rho_{i}(\boldsymbol{x})$, where the summation goes over all defect atoms. This field is calculated on a $20 \times 20 \times 20$ grid and Fig. 9 shows the isosurface of $\rho_{\text {defect }}=0.90$ for the final network of one independent run of each case. It is worth noting that the final networks being compared here also have nearly identical crosslinking degree. Such condition is always maintained when characterizing structure and properties of final networks (e.g. Table 1 \& Figs. 3 - 6. Distribution of defects in the SS case is mostly homogeneous: they appear in small groups that are uniformly found across the domain. In the LM case, clusters of defects are observed and clustering only intensifies in the MM case. This clustering effect is further quantified with the $g(r)$ of defect atoms in Fig. 10, where results from all independent runs are included. Both primary and secondary peaks grow in the order of SS, LM, and MM cases, consistent with images in Fig. 9. Therefore, unlike domain-average structural parameters, the spatial distribution of defects has a strong dependence on precursor topology, which also affects the mechanical properties of the network. Indeed, comparing the observations here with the tensile test results (Figs. 6 \& 7c), it is clear that the extent of defect clustering is correlated with network stiffness: higher clustering is observed in networks with larger apparent modulus. This effect is not observed at the limit of small deformation where the elastic modulus is solely determined by the number density of elastic strands 


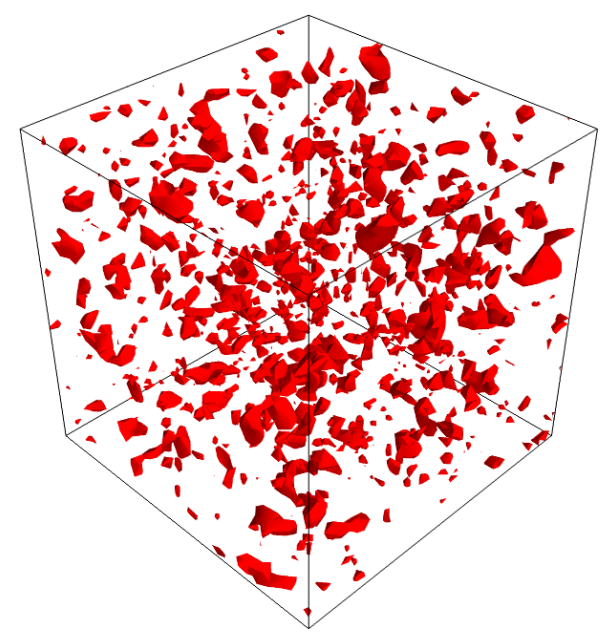

(a)

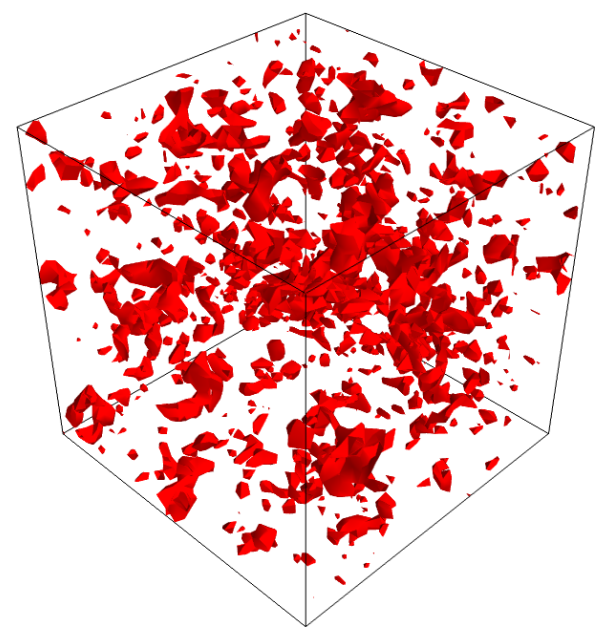

(b)

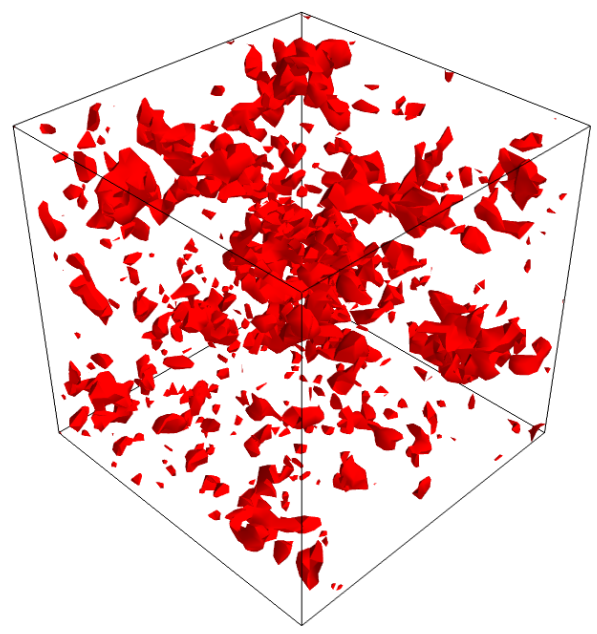

(c)

Figure 9: Density distribution of defect atoms in final network images from selected polymerization runs: (a) SS, (b) LM, and (c) MM.

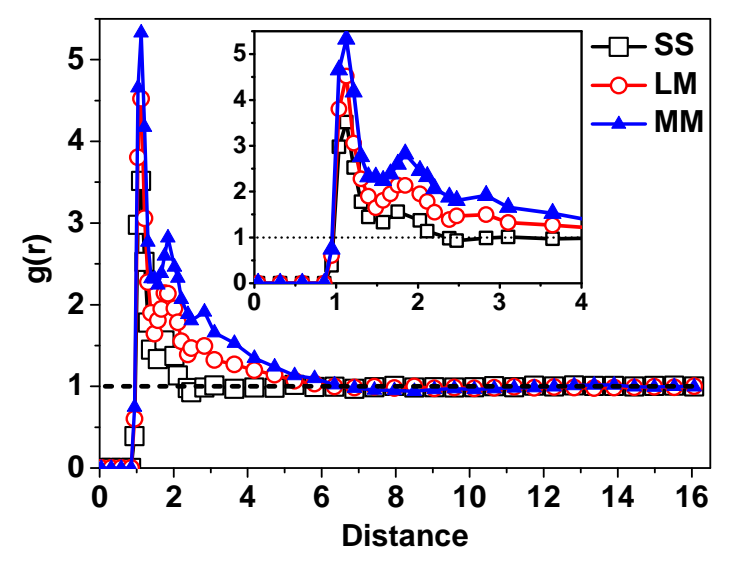

Figure 10: Radial distribution functions of defect atoms in final network images; the inset shows an enlarged view of the small-distance range. Error bars are smaller than the symbol size.

(and thus defects). In the non-linear regime, theoretical prediction is not readily available. In particular, to our best knowledge, there is no existing theory that considers the effect of the spatial distribution of structural features: strands or defects. Indeed, the importance of spatial distribution was not known until this study and further investigation is needed to fully establish the relationship between defect distribution and the network mechanics in the non-linear regime. Meanwhile, we may also draw an analogy with findings in polymer nano-composites, where solid nano-scale particles are blended with a host polymer to reinforce its mechanical strength. During material deformation, stress absorption concentrates around these particles. This reinforcement effect is most effective when particles are uniformly dispersed in the polymer matrix [66]. Similarly, in a polymer network under tensile strain, regions with defects are less capable of absorbing stress which thus weaken its mechanical strength. This weakening effect becomes more pronounced when defect distribution is more uniform, leading to a lower modulus.

We focus instead on the kinetic origin of defect clustering and its precursor dependence. Obviously, precursor topology plays a crucial role in the network formation mechanism. Small-molecule precursors, including both SS and MM cases, first assemble into molecular clusters and gelation occurs when chemical linkages are built between a few major clusters. Meanwhile for the LM case, a few crosslinkers are sufficient to connect the linear chains into a coarse network and form a gel, whose crosslinking density is further increased by "stitching up" the space between these linkage points. However, our observation that in terms of both defect clustering and mechanical stiffness, the LM network is ranked between the SS and MM ones cannot be explained by this difference in the gelation pathway. In another direction of thought, the MM and LM cases both contain monomeric precursors whose diffusion is relatively unconstrained, whereas the SS case contains short-chain precursors only whose slow diffusion, especially after the gel point, can result in significant kinetic trapping. This would however predict a higher extend of non-uniformity in the MM case, opposite to our observation.

Realizing that topological defects only occur around atoms whose bonding sites are not fully occupied, we plot the density distribution of these unsaturated atoms (only including those on the gel - the biggest molecule in the system) in Fig. 11 for the final networks (one independent run for each case). These density fields are generated in the same way as Fig. 9 but an isosurface level of 0.35 is shown. It is clear that for both LM and MM systems, unsaturated atoms of opposite types accumulate to separate localized regions. Since these atoms are topologically constrained in the network by chemical bonds, further elimination of these defects is nearly impossible and large regions of clustered defects are left. By contrast, unsaturated atoms of both types are uniformly distributed in the 


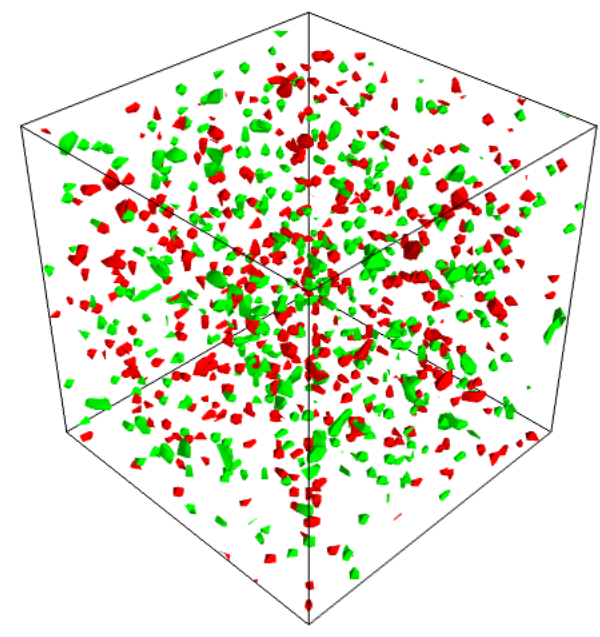

(a)

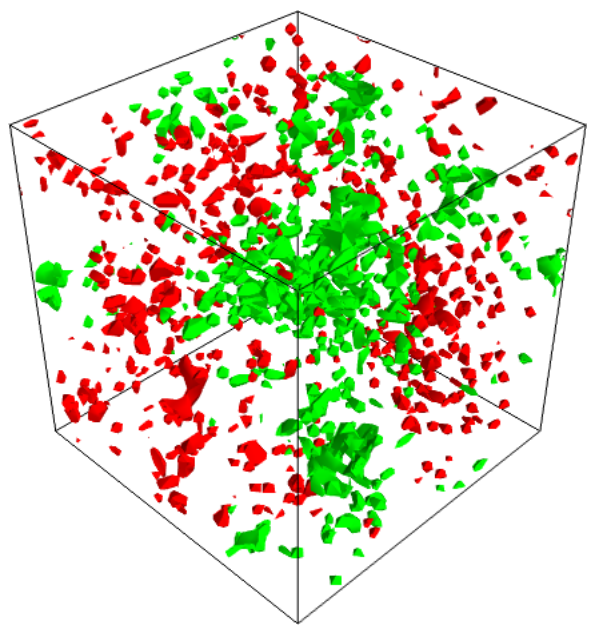

(b)

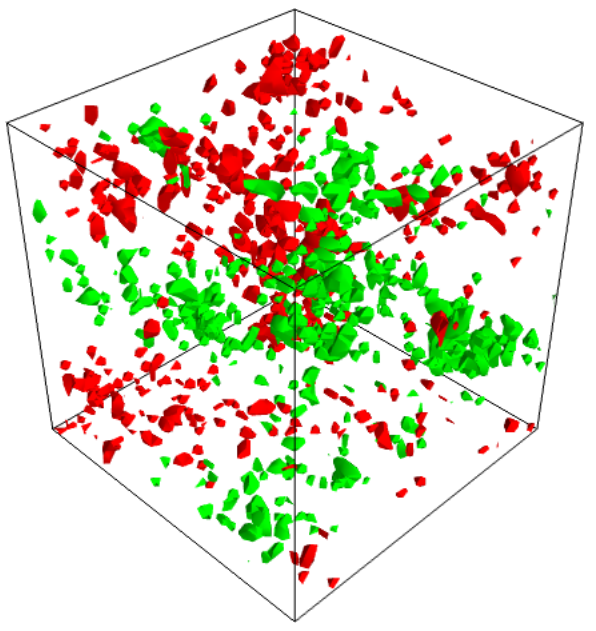

(c)

Figure 11: Density distribution of unsaturated atoms on the network in final network images from selected polymerization runs: (a) SS, (b) LM, and (c) MM. Green (light): trifunctional atoms; red (dark): bifunctional atoms.

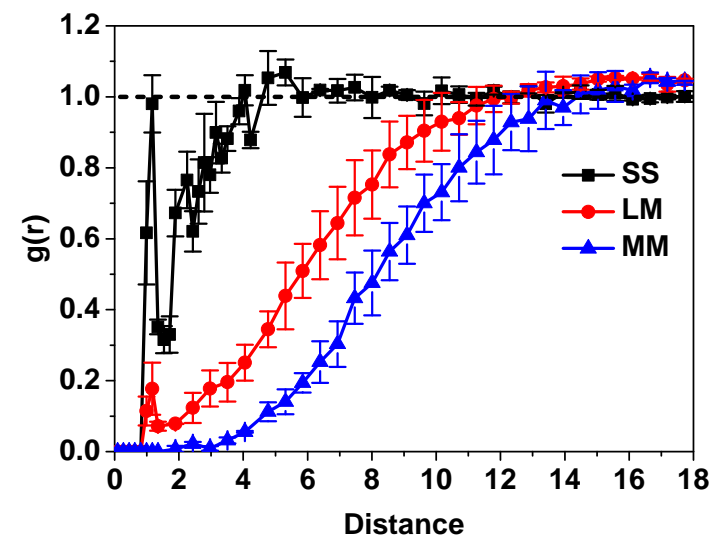

Figure 12: Radial distribution functions of unsaturated trifunctional atoms using unsaturated bifunctional atoms as references in final network images. For both atom types, only those on the network are included.

SS network, leaving only small spots of defects. This observation is confirmed with the $g(r)$ between unsaturated atoms of opposite types. Using unsaturated bifunctional atoms as references, profiles in Fig. 12 are proportional to the density of unsaturated trifunctional atoms at given distances. Compared with the LM and MM cases, where a layer depleted of atoms of opposite type for potential bonding exists near most unsaturated atoms, there is a much better chance for unsaturated atoms in the SS case to find a nearby bonding partner.

Local disparity of unsaturated atoms increases in the order of SS, LM, and MM cases, which is not only consistent with increasing defect clustering and mechanical stiffness observed above, but also predicts that defects in the LM and MM cases are harder to eliminate than those in the SS case. This is confirmed in Fig. 13 where the time evolution of defect atoms in the network (i.e., the largest cluster in the domain) is compared between different pre-

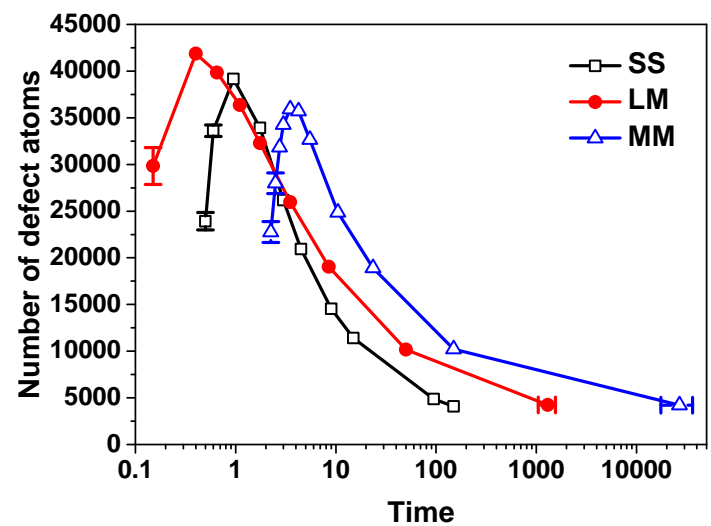

Figure 13: Time evolution of structural defects during the polymerization processes. Error bars smaller than symbol size are not shown.

cursor systems. The profiles initially rise up because the number of defect atoms increases with the increasing total number of polymerized atoms. A peak is reached shortly after the gel point (cf. Fig. 2b), after which the reaction is dominated by defect elimination. Although the LM case is the quickest in reaching gelation, the rate of defect elimination is 10 times higher in the SS case, which is two orders of magnitude higher than that of the MM case.

The reason for this spatial separation between atoms of opposite types in the LM and MM case, and the lack thereof in the SS case, becomes clear when we look at the atom molar ratio distribution during the polymerization process in Fig. 14. Here, we divide the simulation domain into a uniform $5 \times 5 \times 5$ grid and the molar ratio between bifunctional and trifunctional atoms are calculated in each cell. Only atoms chemically bonded to multi-atom molecules are counted, since single atoms are free to move in the space and thus not expected to have a lasting effect on the local molar ratio. The color scale shows the 


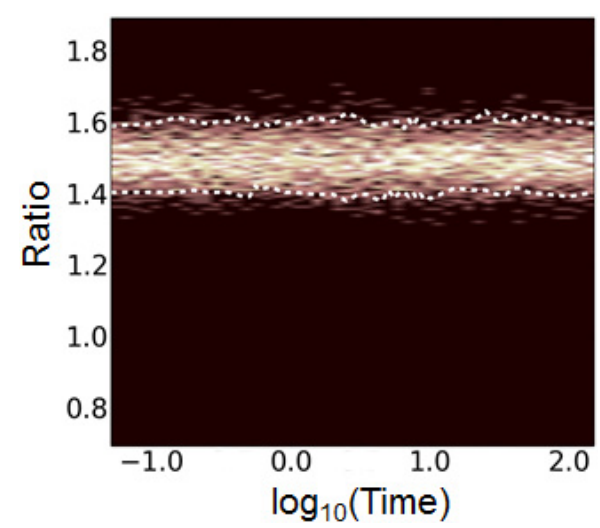

(a)

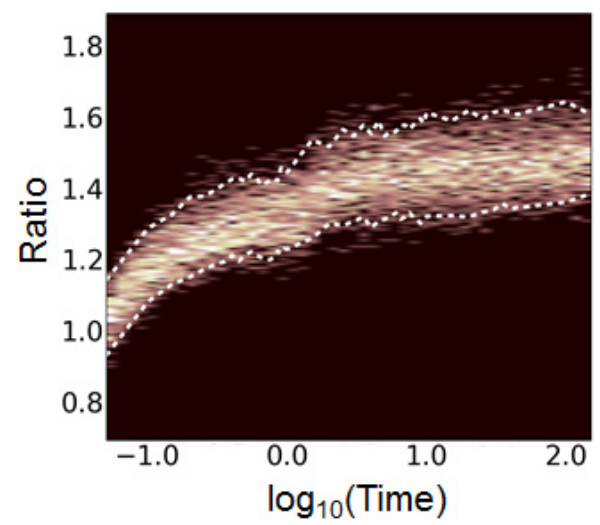

(b)

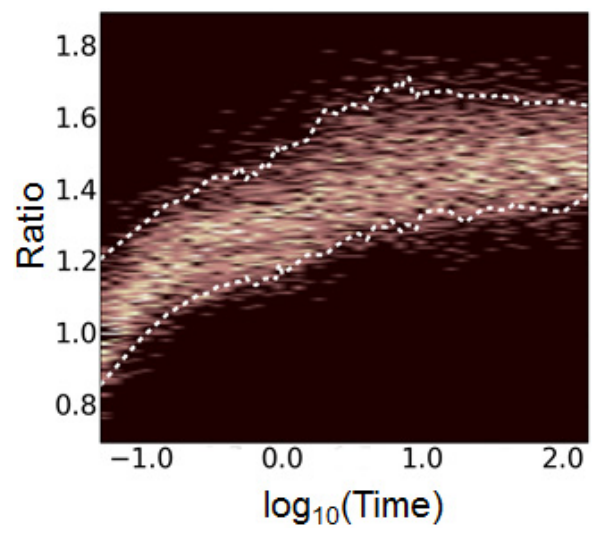

(c)

Figure 14: Time evolution of the spatial distribution of the molar ratio between bonded bifunctional atoms and bonded trifunctional atoms: (a) SS, (b) LM and (c) MM. The color scale is proportional to the probability density of finding a region with a certain molar ratio at the given time: bright - high; dark - low. White dashed lines show the molar-ratio range covering $95 \%$ of the domain.

probability density of each molar ratio value at the given time. Note that with the overall atom ratio fixed at $3: 2$ between the two types, all cases have to converge to an average of 1.5 as the network completes. For the SS case, the molar ratio is around 1.5 throughout the polymerization process, which is a direct consequence of the precursor design: each precursor molecule has exactly 3 bifunctional and 2 trifunctional atoms and thus the $3: 2$ ratio is maintained across the domain. The LM case starts with a ratio around 1, a reflection of the $99: 100$ ratio in the polymeric precursors. It gradually increases to 1.5 as additional bifunctional crosslinkers connect with the polymer. The MM case also starts near 1 because at the beginning, each time a new bond is formed, it creates a dimer containing one atom of either type. Note that the reaction stoichiometry requires a ratio of $3: 2$ for complete conversion. Comparing all three cases, the SS case not only maintains this ratio throughout the whole process, variation between different spatial regions (cells) is also small. Meanwhile, from the LM case to the MM one, spatial variation of the molar ratio substantially increases, indicating that there is a better chance of having local surplus of a certain atom type. Higher spatial variation in these two cases is not surprising, considering, for example, that the MM case allows the highest degree of freedom, i.e., more randomness, for the assembly of atoms into molecules. This large variation eventually leads to the difficulty of defect elimination at certain regions and thus defect clustering.

\section{Conclusion}

Formation of a model polymer network is studied using CG MD simulation starting from three different sets of precursors. The precursors are designed such that at the ideal limit of perfect conversion, they would result in an identical network structure. At the same level of atom saturation (and thus crosslinking density), networks generated from different precursor systems show little differ- ence in domain-average statistics of network connectivity. Other commonly examined properties, including the radial distribution function and the glass transition temperature, also show no discernible dependence on the precursor choice. Precursor effects are however clearly observed in the mechanical properties of polymer networks: although the stress-strain curves overlap one another in the linear regime of deformation, at larger deformation a clear dependence on the precursor choice is observed, where networks formed from SS precursors are more ductile than those from individual monomers (MM). Compared with other structural parameters, the number of elastic strands is found to best correlate with the elastic modulus (in the non-linear regime). This correlation however breaks down near the limit of full conversion, where the elastic modulus can change by $\approx 30 \%$ for the same elastic-strand density, depending on the precursor choice. This change is found to correlate with the spatial distribution of topological defects and a higher degree of defect clustering leads to higher network stiffness. The origin of defect clustering roots in the precursor design. The SS case, which essentially pre-assembles constituting monomers into smallmolecule precursors with the exact stoichiometric ratio, maintains this ratio in different spatial regions, leading to not only uniform network structure but also faster defect elimination. From the LM case to the MM case, increasing randomness in the monomer assembling process leads to larger variation in local atom molar ratios, resulting in defect clustering and slower defect elimination.

There are several important takeaways from these findings especially for the development of molecular models of polymer networks. First, the choice of precursors has lasting impact on the structure and properties of polymer networks. It is therefore important to design a virtual synthesis pathway that resembles the realistic crosslinking process in experiments, for which CG simulation followed by reserve mapping will be necessary to get a full-atom model. Second, validation of network models needs to con- 
sider defect distribution, in addition to typical characterizations currently used in the literature. Finally, when the goal is to maximize the network uniformity and minimize the residual defects, it is advantageous to prepare precursors with the required stoichiometric ratio of monomers, before the start of the simulated polymerization process.

\section{Acknowledgment}

The authors gratefully acknowledge the financial support from the Natural Sciences and Engineering Research Council of Canada (NSERC) through its Discovery Grants Program (No. RGPIN-2014-04903) as well as the computing resource allocated by Compute/Calcul Canada. This work is made possible by the facilities of the Shared Hierarchical Academic Research Computing Network (SHARCNET: www.sharcnet.ca).

\section{References}

1. Aharoni SM. Synthesis, characterization, and theory of polymeric networks and gels. Springer; 1992.

2. Dušek K. Polymer networks. Berlin Heidelberg: Springer; 1992.

3. Seiffert S, Sprakel J. Physical chemistry of supramolecular polymer networks. Chemical Society Reviews 2012;41(2):909-30.

4. Nouri N, Ziaei-Rad S. A molecular dynamics investigation on mechanical properties of cross-linked polymer networks. Macromolecules 2011;44(13):5481-9.

5. Varshney V, Patnaik SS, Roy AK, Farmer BL. A molecular dynamics study of epoxy-based networks: cross-linking procedure and prediction of molecular and material properties. Macromolecules 2008;41(18):6837-42.

6. Krumova M, Lopez D, Benavente R, Mijangos C, Perena J. Effect of crosslinking on the mechanical and thermal properties of poly (vinyl alcohol). Polymer 2000;41(26):9265-72.

7. Yagyu H, Utsumi T. Coarse-grained molecular dynamics simulation of nanofilled crosslinked rubber. Computational Materials Science 2009;46(2):286-92.

8. Wu C, Xu W. Atomistic molecular modelling of crosslinked epoxy resin. Polymer 2006;47(16):6004-9.

9. Izumi A, Nakao T, Shibayama M. Atomistic molecular dynamics study of cross-linked phenolic resins. Soft Matter 2012;8(19):5283-92.

10. Zhang S, So LLC, Faucher S, Xi L. Polymer coating over solid particles with in situ thermal curing. Ind Eng Chem Res 2016;55:5574-84. doi \{10.1021/acs.iecr.6b00376\}

11. Seliktar D. Designing cell-compatible hydrogels for biomedical applications. Science 2012;336:1124-8. doi 10.1126/science. 1214804

12. Knop A, Pilato LA. Phenolic resins: chemistry, applications and performance. Springer Science \& Business Media; 2013.

13. Binder K. Monte Carlo and molecular dynamics simulations in polymer science. Oxford University Press; 1995.

14. Grest GS, Kremer K. Molecular dynamics simulation for polymers in the presence of a heat bath. Physical Review A 1986;33(5):3628.

15. Glotzer SC, Paul W. Molecular and mesoscale simulation methods for polymer materials. Ann Rev Mater Res 2002;32:401-36. doi $\{10.1146 /$ annurev.matsci.32.010802.112213\}

16. Paul W, Smith GD. Structure and dynamics of amorphous polymers: Computer simulations compared to experiment and theory. Rep Prog Phys 2004;67:1117-85.

17. Theodorou DN, Suter UW. Detailed molecular-structure of a vinyl polymer glass. Macromolecules 1985;18:1467-78. doi 10 . $1021 / \mathrm{ma} 00149 \mathrm{a} 018$
18. Carbone P, Karimi-Varzaneh HA, Müller-Plathe F. Finegraining without coarse-graining: an easy and fast way to equilibrate dense polymer melts. Faraday Discuss 2010;144:25-42. doi $\{10.1039 /$ b902363a $\}$

19. Sliozberg YR, Andzelm JW. Fast protocol for equilibration of entangled and branched polymer chains. Chemical Physics Letters 2012;523:139-43.

20. Abbott LJ, Hart KE, Colina CM. Polymatic: a generalized simulated polymerization algorithm for amorphous polymers. Theor Chem Acc 2013;132:1334. doi \{10.1007/ s00214-013-1334-z\}

21. Hörstermann H, Hentschke R, Amkreutz M, Hoffmann M, Wirts-Ruetters M. Predicting water sorption and volume swelling in dense polymer systems via computer simulation. J Phys Chem B 2010;114:17013-24. doi \{10.1021/jp105210y\}

22. Khare KS, Khare R. Directed diffusion approach for preparing atomistic models of crosslinked epoxy for use in molecular simulations. Macromol Theory Simul 2012;21:322-7. doi $\{10$. 1002/mats. 201100119\}

23. Abbott LJ, Hughes JE, Colina CM. Virtual synthesis of thermally cross-linked copolymers from a novel implementation of Polymatic. J Phys Chem B 2014;118:1916-24. doi 10.1021/ jp409664d

24. Lin PH, Khare R. Molecular simulation of cross-linked epoxy and epoxy-POSS nanocomposite. Macromolecules 2009;42:4319-27. doi \{10.1021/ma9004007\}.

25. Jang C, Sirk TW, Andzelm JW, Abrams CF. Comparison of crosslinking algorithms in molecular dynamics simulation of thermosetting polymers. Macromol Theory Simul 2015;24:26070. doi \{10.1002/mats.201400094\}

26. Varshney V, Patnaik SS, Roy AK, Farmer BL. A molecular dynamics study of epoxy-based networks: Cross-linking procedure and prediction of molecular and material properties. Macromolecules 2008;41:6837-42. doi \{10.1021/ma801153e\}.

27. Demir B, Walsh TR. A robust and reproducible procedure for cross-linking thermoset polymers using molecular simulation. Soft Matter 2016;12:2453-64. doi 10.1039/c5sm02788h.

28. Leung YK, Eichinger B. Computer simulation of end-linked elastomers. i. trifunctional networks cured in the bulk. The Journal of chemical physics 1984;80(8):3877-84.

29. Leung YK, Eichinger B. Computer simulation of end-linked elastomers. ii. bulk cured tetrafunctional networks. The Journal of chemical physics 1984;80(8):3885-91.

30. Duering ER, Kremer K, Grest GS. Dynamics of model networks: the role of the melt entanglement length. Macromolecules 1993;26(12):3241-4.

31. Duering ER, Kremer K, Grest GS. Structure and relaxation of end-linked polymer networks. The Journal of chemical physics 1994;101(9):8169-92.

32. Grest GS, Kremer K, Duering ER. Kinetics and relaxation of end crosslinked polymer networks. Physica A: Statistical Mechanics and Its Applications 1993;194(1):330-7.

33. Tsige M, Lorenz CD, Stevens MJ. Role of network connectivity on the mechanical properties of highly cross-linked polymers. Macromolecules 2004;37(22):8466-72.

34. Yang W, Wei D, Jin X, Liao Q. Molecular dynamics simulation of the formation of polymer networks. Macromolecular theory and simulations 2007;16(5):548-56.

35. Hosono N, Masubuchi Y, Furukawa H, Watanabe T. A molecular dynamics simulation study on polymer networks of endlinked flexible or rigid chains. The Journal of chemical physics 2007;127(16):164905.

36. Sliozberg YR, Chantawansri TL. Computational study of imperfect networks using a coarse-grained model. The Journal of chemical physics 2013;139(19):194904.

37. Tsige M, Stevens MJ. Effect of cross-linker functionality on the adhesion of highly cross-linked polymer networks: a molecular dynamics study of epoxies. Macromolecules 2004;37(2):630-7.

38. Liu J, Cao D, Zhang L. Static and dynamic properties of model elastomer with various cross-linking densities: A molecular dynamics study. J Chem Phys 2009;131:034903. doi \{10.1063/1. 


\section{1\}}

39. Liu J, Zhang L, Cao D, Shen J, Gao Y. Computational simulation of elastomer nanocomposites: current progress and future challenges. Rubber Chem Technol 2012;85:450-81. doi \{10. 5254/rct.12.87966\}

40. Yin DW, Yan QL, de Pablo JJ. Molecular dynamics simulation of discontinuous volume phase transitions in highly-charged crosslinked polyelectrolyte networks with explicit counterions in good solvent. J Chem Phys 2005;123:174909. doi \{10.1063/1. 2102827\}.

41. Stevens MJ. Interfacial fracture between highly cross-linked polymer networks and a solid surface: effect of interfacial bond density. Macromolecules 2001;34(8):2710-8.

42. Carmesin I, Kremer K. The bond fluctuation method: a new effective algorithm for the dynamics of polymers in all spatial dimensions. Macromolecules 1988;21:2819-23. doi 10.1021/ ma00187a030

43. Gilra GN, Cohen C, Panagiotopoulos AZ. A Monte Carlo study of the structural properties of end-linked polymer networks. $J$ Chem Phys 2000;112:6910-6. doi \{10.1063/1.481264\}

44. Izvekov S, Voth GA. Multiscale coarse graining of liquidstate systems. J Chem Phys 2005;123:134105. doi:10.1063/ 1.2038787.

45. Peter C, Kremer K. Multiscale simulation of soft matter systems - from the atomistic to the coarse-grained level and back. Soft Matter 2009;5(22):4357-66.

46. de Pablo JJ. Coarse-Grained Simulations of Macromolecules: From DNA to Nanocomposites. Annu Rev Phys Chem 2011;62:555-74. doi \{10.1146/ annurev-physchem-032210-103458\}

47. Yang S, Cui Z, Qu J. A coarse-grained model for epoxy molding compound. J Phys Chem B 2014;118:1660-9. doi:10.1021/ jp409297t

48. Komarov PV, Chiu YT, Chen SM, Khalatur PG, Reineker P. Highly cross-linked epoxy resins: an atomistic molecular dynamics simulation combined with a mapping/reverse mapping procedure. Macromolecules 2007;40:8104-13. doi \{10.1021/ ma070702\}

49. Liu H, Li M, Lu ZY, Zhang ZG, Sun CC, Cui T. Multiscale simulation study on the curing reaction and the network structure in a typical epoxy system. Macromolecules 2011;44:8650-60. doi $\{10.1021 / \mathrm{ma} 201390 \mathrm{k}\}$

50. Groot RD, Warren PB. Dissipative particle dynamics: Bridging the gap between atomistic and mesoscopic simulation. J Chem Phys 1997;107:4423-35. doi 10.1063/1.474784

51. Kremer K, Grest GS. Dynamics of entangled linear polymer melts: A molecular-dynamics simulation. The Journal of Chemical Physics 1990;92(8):5057-86.

52. Frenkel D, Smit B. Understanding Molecular Simulation: from Algorithms to Applications. 2nd ed.; London: Academic Press; 2002.

53. Plimpton S. Fast parallel algorithms for short-range moleculardynamics. J Comput Phys 1995;117:1-19. URL: http:// lammps.sandia.gov doi 10.1006/jcph.1995.1039

54. Auhl R, Everaers R, Grest GS, Kremer K, Plimpton SJ. Equilibration of long chain polymer melts in computer simulations. The Journal of chemical physics 2003;119(24):12718-28.

55. Shinoda W, Shiga M, Mikami M. Rapid estimation of elastic constants by molecular dynamics simulation under constant stress. Phys Rev B 2004;69:134103. doi 10.1103/PhysRevB.69. 134103

56. Balabanyan A, Kramarenko EY, Ronova I, Khokhlov A. Monte carlo study of structure and kinetics of formation of end-linked polymer networks. Polymer 2005;46(12):4248-57.

57. Duering E, Kremer K, Grest G. Structural properties of randomly crosslinked polymer networks. In: Wartewig S, Helmis G, eds. Physics of Polymer Networks; vol. 90 of Progr. Colloid Polym. Sci. Darmstadt: Springer; 1992:13-5.

58. Herrmann H, Hong D, Stanley H. Backbone and elastic backbone of percolation clusters obtained by the new method of 'burning'. J Phys A Math Gen 1984;17:L261-6. doi 10.1088/
0305-4470/17/5/008

59. Makke A, Perez M, Rottler J, Lame O, Barrat JL. Predictors of Cavitation in Glassy Polymers under Tensile Strain: A CoarseGrained Molecular Dynamics Investigation. Macromol Theory Simul 2011;20:826-36. doi 10.1002/mats.201100006

60. Morita H, Tanaka K, Kajiyama T, Nishi T, Doi M. Study of the glass transition temperature of polymer surface by coarse-grained molecular dynamics simulation. Macromolecules 2006;39:6233-7. doi 10.1021/ma052632h

61. Shavit A, Riggleman RA. Influence of backbone rigidity on nanoscale confinement effects in model glass-forming polymers. Macromolecules 2013;46:5044-52. doi 10.1021/ma400210w

62. Soni NJ, Lin PH, Khare R. Effect of cross-linker length on the thermal and volumetric properties of cross-linked epoxy networks: A molecular simulation study. Polymer 2012;53:1015-9. doi $10.1016 / \mathrm{j} \cdot$ polymer.2011.12.051

63. Rubinstein M, Colby RH. Polymer Physics. New York: Oxford University Press; 2003.

64. Larsen AL, Hansen K, Sommer-Larsen P, Hassager O, Bach A, Ndoni S, Jørgensen M. Elastic properties of nonstoichiometric reacted pdms networks. Macromolecules 2003;36(26):10063-70.

65. Willard AP, Chandler D. Instantaneous liquid interfaces. $J$ Phys Chem B 2010;114:1954-8. doi 10.1021/jp909219k

66. Zheng Y, Zheng Y, Ning R. Effects of nanoparticles $\mathrm{SiO}_{2}$ on the performance of nanocomposites. Mater Lett 2003;57:29404. doi $10.1016 / \mathrm{S} 0167-577 \mathrm{X}(02) 01401-5$ 